\title{
EIMERIA TENELLA INFECTION MODULATES THE EXPRESSION LEVELS OF INTESTINAL EPITHELIAL BARRIER-RELATED GENES IN CHICKEN
}

\author{
Hung Hoang Son PHAM${ }^{1}$, Toshimitsu HATABU²* \\ ${ }^{1}$ Graduate School of Environmental and Life Science, Okayama University \\ (1-1-1, Tsushima-Naka, Kita-Ku, Okayama 700-8530, Japan) \\ E-mail: phamhoangsonhung@huaf.edu.vn \\ 2* Correspondence: Associate Professor, Graduate School of Environmental and Life Science, Okayama University \\ (1-1-1 Tsushima-Naka, Kita-Ku, Okayama 700-8530, Japan) \\ E-mail: hatabu@okayama-u.ac.jp.
}

\begin{abstract}
Eimeria tenella infection causes coccidiosis, which induces diarrhea and bloody stool in chicken. This infection impairs the epithelial barrier that lines the intestinal tract. The intercellular junctions are key factors for maintaining barrier integrity. However, information on the gene expression modulation of junctional molecule-related genes is still limited. This study aimed to elucidate the association between disease symptoms and the regulation of target genes. Intestinal function was evaluated using biomarker measurements. Gene expression regulation was determined by real-time quantitative polymerase chain reaction. The disruption of the intestinal barrier might be induced by lower levels of claudin-1, claudin-3, E-cadherin, occludin, and ZO-1 but higher levels of claudin-2. Our results might suggest the association between the modulation of junctional barrier molecules and major symptoms of $E$. tenella infection.
\end{abstract}

Key Words: bloody stool, diarrhea, Eimeria tenella, epithelial barrier, junctional molecules

\section{INTRODUCTION}

Coccidiosis caused by Eimeria tenella is a serious intestinal disease in chickens. Diarrhea and bloody stool are the major symptoms of this disease. Infected chickens experience depressed feed consumption, weight loss, and decreased performance. Coccidiosis has become a large concern in the worldwide poultry industry because of expensive investments in disease prevention and treatment. More seriously, in settings of a high mortality rate, loss of production aggravates economic distress ${ }^{1}$.

E. tenella is an obligate intracellular protozoan parasite with a complex life cycle, consisting of an exogenous phase in the environment and an endogenous phase in the host intestine. In the exogenous phase, unsporulated oocysts are released from chicken droppings and undergo sporulation at the right temperature, humidity, and oxygen level. Subsequently, oocysts, which form four sporocysts that are covered with two sporozoites, are considered to be infectious. The endogenous stage begins when a chicken ingests an oocyst through drinking or eating off the ground contaminated with feces from other infected chicks. When the sporozoites attach themselves to the intestinal lining, the parasites penetrate into the epithelial barrier. Epithelial cells are destroyed after several rounds of asexual and sexual reproduction. Finally, fertilization and formation of unsporulated oocysts create the next generation of oocysts ${ }^{2)}$.

The chicken intestine is lined by an epithelial barrier, which is of great importance to food digestion and nutrient absorption. The integrity of the intestinal epithelial barrier is sustained by the balance of the intercellular junction molecule complexes. These molecules are crucial for the regulation of the paracellular passage, which prevents proinflammation molecules from penetrating the mucosal tissue ${ }^{3)}$. However, understanding of the gene expression modulation of the intestinal epithelial barrier in the chicken with E. tenella infection is limited. Thus, this study aims to identify the correlation between symptoms and the regulation of junctional molecular genes involved in the intestinal epithelial barrier.

\section{MATERIALS AND METHODS}

(1) Parasite, animals, and experimental design

White Leghorn chickens $(n=45)$ were randomly 
divided into the following three groups: uninfected (control), E. tenella, and a fasting group (feed withdrawn 24 hours before processing). Chickens were maintained in a coccidia-free environment. All procedures were approved by the Animal Care and Use Committee, Okayama University (OKU-2018561) and were conducted in accordance with the Policy on the Care and Use of the Laboratory Animals, Okayama University.

At 14 days of age, chickens in the infected group were orally administered $1 \times 10^{4}$ oocysts/chick of mature sporulated oocysts of the E. tenella NIAH strain, which was maintained at the Laboratory of Animal Physiology in Okayama University (Okayama, Japan). Three chicks from each group were sacrificed via cervical dislocation for cecum collection. One piece of cecum was immediately frozen at $-80{ }^{\circ} \mathrm{C}$ for gene expression analysis, and another piece was fixed with $10 \%$ buffered formalin for histopathological assay.

\section{(2) Fecal collection and oocyst counting}

To count oocysts, feces were monitored daily from 5 to 10 days postinfection (dpi). Oocysts per gram of feces were identified by the fecal flotation method using the saturated sucrose solution.

\section{(3) Histopathological observation}

The paraffin-embedded tissue block was sectioned at a thickness of $6 \mu \mathrm{m}$ and deparaffinized. Sectioned specimens were stained using a hematoxylin-eosin (HE) solution. To evaluate the inflammation levels of ceca, histopathological observation was performed under a digital optical microscope (Olympus FSX100, Olympus, Tokyo, Japan). We used an inflammation scoring system ranging from 0 to 4 , which was modified according to the method described previously ${ }^{4}$.

\section{(4) Measurement of intestinal permeability}

The plasma concentration of fluorescein isothiocyanate-dextran (FITC-d; molecular weight 4000 Da; Sigma-Aldrich Co., St. Louis, MO, USA) was determined according to the method of Kuttappan et al. ${ }^{5)}$ The fluorescence concentration in plasma was evaluated using a Multimode Microplate Reader (SH-9000 Serial, Corona Electric Co., Ltd) with an excitation of $485 \mathrm{~nm}$ and an emission wavelength of $528 \mathrm{~nm}$. Levels of fluorescence in the samples were converted to respective FITC-d microgram per milliliter.

\section{(5) Gene expression analysis}

We used RNAzol RT Reagent (COSMO BIO Co., Ltd., Tokyo, Japan) to extract total RNA from the ceca following the manufacturer's instructions.
Complementary DNAs were synthesized using $1 \mu \mathrm{g}$ of total RNA as the template, with oligo dT primer and the ReverTra Ace Master Mix kit (Toyobo Co., Ltd, Osaka, Japan).

We performed quantitative real-time polymerase chain reaction using the Mini Opticon Real-Time PCR System (Bio-Rad Laboratories Inc., Hercules, CA, USA) with the Brilliant III Ultra-Fast SYBR ${ }^{\circledR}$ Green QPCR Master Mix (Agilent Technologies, West Cedar Creek, TX, USA). The relative mRNA level was estimated by the comparative CT method $^{6}$.

\section{(6) Statistical analysis}

Experiment data are represented by at least three repeat experiments. We statistically analyzed data using one-way analysis of variance with Tukey's multiple comparison test; results are presented as mean \pm standard error of the mean.

\section{RESULTS}

\section{(1) Oocyst shedding and gross observation}

Evaluation of the number of oocysts in feces indicated that the oocysts began shedding at $6 \mathrm{dpi}$ and reached a maximum at 7 dpi (data not shown). In the E. tenella group, all chickens had diarrhea from 3 to 7 dpi. Diarrhea with blood occurred from 4 to $6 \mathrm{dpi}$ (Table 1). In all uninfected chickens, the cecum had a smooth and glossy margin, filled with feces and without bleeding or enteritis. In contrast, the cecum of infected chickens showed atrophy as a result of dehydration. Mucus and clotted blood were observed from 4 to 6 dpi in the lumen of the cecum (data not shown).

\section{(2) Histopathological examination}

The epithelial monolayer was normal until 5 dpi (Fig. 1A-C) but became detached at 6 dpi (Fig. 1D). HE-stained cecum specimens indicated villous atrophy, severe inflammation, hemorrhage, a proliferation of epithelial cells around intestinal crypts, and epithelial desquamation. The lesion score increased to the maximum of $3.78 \pm 0.11$ at $7 \mathrm{dpi}$ (Table 1).

\section{(3) FITC-d levels in plasma}

During the experiment, the plasma FITC-d level in the E. tenella group was persistently significantly

Table 1. Observation of feces and evaluation of histomorphology lesion score.

\begin{tabular}{cccccccc}
\hline DPI & $\mathbf{2}$ & $\mathbf{3}$ & $\mathbf{4}$ & $\mathbf{5}$ & $\mathbf{6}$ & $\mathbf{7}$ & $\mathbf{8}$ \\
\hline Blooding & - & - & + & + & + & - & - \\
Diarrhea & - & + & + & + & + & + & - \\
\hline \multirow{2}{*}{ Lesion } & $0.83^{\mathrm{a}}$ & $1.22^{\mathrm{a}}$ & $2.44^{\mathrm{b}}$ & $3.5^{\mathrm{c}}$ & $3.56^{\mathrm{c}}$ & $3.78^{\mathrm{c}}$ & $3.67^{\mathrm{c}}$ \\
score & \pm & \pm & \pm & \pm & \pm & \pm & \pm \\
& 0.17 & 0.11 & 0.22 & 0.10 & 0.11 & 0.11 & 0.19 \\
\hline
\end{tabular}

${ }^{\mathrm{a}-\mathrm{c}}$ Different superscripts indicate statistical differences between dpi $(p<0.05)$. 
higher than that of the control group. In particular, the concentration of FITC-d in the blood of $E$. tenella-infected chickens was sharply increased at 5 dpi (Fig. 2). However, the plasma FITC-d levels were not elevated in the fasting and control groups throughout the different time courses of the experiment.

\section{(4) mRNA expression in cecum with $E$. tenella}

The comparison between the control and infected groups indicated that, in the latter group, the relative mRNA level of claudin-2 (CLDN-2) was significantly upregulated in the cecum at 3, 4, and 5 dpi (Fig. 3B). In contrast, the mRNA levels of CLDN-3, E-cadherin (E-cad), occludin (OCDN), and ZO-1 were noticeably reduced (Fig. 3C, D, E, F). Interestingly, CLDN-1 mRNA levels were initially decreased at 3 and 4 dpi but then increased at 5 and 6 dpi (Fig. 3A).

\section{DISCUSSION}

A single layer of stacked columnar epithelial cells lines the intestine and is the key factor in the maintenance of epithelial barrier integrity. In this layer, the extended lateral surfaces of adjacent cells form close contacts via apical junctional complexes, called the tight and adherens junctions ${ }^{7}$.

Claudin proteins are the main components of the tight junctions, and they form a seal to modulate paracellular transport in the intestinal epithelium. It is known that CLDN-1 and CLDN-3 are sealing claudins, the increased expression of which leads to a tight epithelium ${ }^{8}$. In this study, we recorded a notable downregulation of CLDN-3 mRNA level in E. tenella-infected chicks as compared with uninfected chicks. Reports showed that a low level of sealing proteins was the reason for the epithelial barrier leakage and detrimental movement of luminal contents through the paracellular space ${ }^{9)}$. Similarly, we noted a downregulated tendency in CLDN-1 expression from 2 to $4 \mathrm{dpi}$, which contributed to the barrier dysfunction, leading to diarrhea and bloody feces. However, at $5 \mathrm{dpi}$, there was a sharp increase in the gene expression of CLDN-1, which could be associated with the recovery mechanism at the intestinal epithelium to restore barrier function and return to homeostatic functioning. Further experiments should be conducted in the future to clarify this speculation. In contrast to sealing claudins, pore-forming proteins such as CLDN-2 have higher expressions that facilitate paracellular permeability by allowing more luminal contents to translocate to the systemic circulation. CLDN-2 proteins, which have the ability to form paracellular pores and water channels, give rise to leaky epithelia once they are predominantly expressed, by allowing

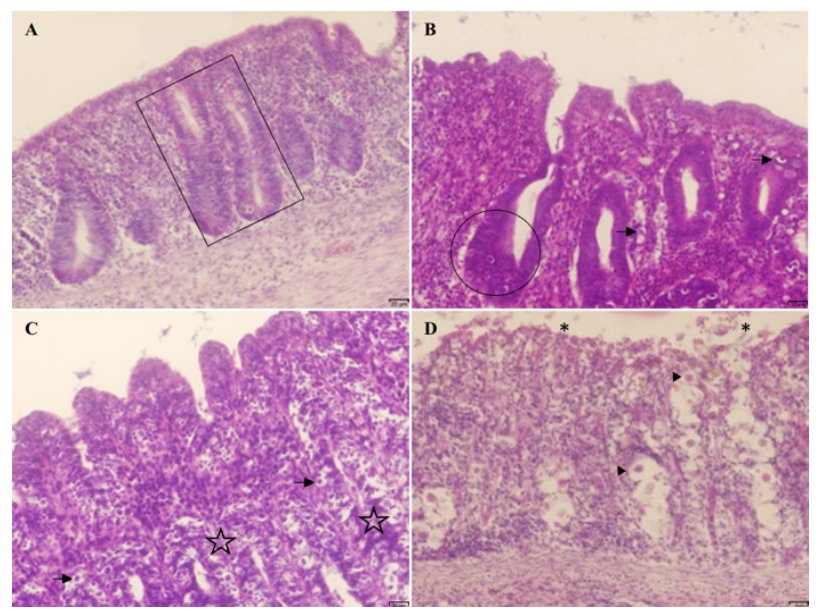

Fig 1. Photomicrograph of the cecum of E. tenella-infected chickens showing hyperplasia of the intestinal crypts (square) at 3 dpi (A). The presence of coccidia schizont (arrows) associated with a proliferation of epithelial cells (cycle) around crypts at 4 dpi (B). Heavy infestation of crypts (stars) and schizonts (arrows) lining the epithelium at $5 \mathrm{dpi}$ (C). Abundance of oocysts (arrowheads) in the lamina propria, with inflammation and epithelial desquamation (asterisks) at $6 \mathrm{dpi}$ (D).

the passage of solute ions. An increased level of CLDN-2 is also responsible for inflammation and infection $^{10)}$. In our study, the level of CLDN-2 mRNA was especially higher in chicks infected with E. tenella than in noninfected chicks. In addition, the parasitic infection induced a remarkable upregulation of CLDN-2 mRNA expression from 3 to 5 dpi. Consequently, a higher level of FITC-d in blood was found at $3 \mathrm{dpi}$, with the highest level recorded at 5 dpi, indicating an increasing absorption via the disrupted intestinal barrier, which resulted in the diarrhea as observed from $3 \mathrm{dpi}$.

Another important component of the tight junction barrier is OCDN, which involves the regulation of paracellular transport and leaky pathways ${ }^{11)}$. In our study, the mRNA expression level of OCDN in the infected chickens was significantly lower than in controls at 4 and 5 dpi. In agreement with our result, Cani et al. showed that the downregulated expression of OCDN coincided with the onset of diarrhea and the increased plasma level of FITC-d ${ }^{12)}$. This

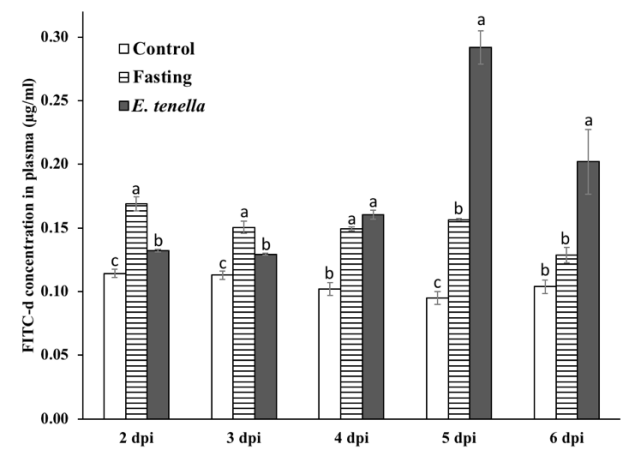

Fig 2. Plasma FITC-d levels in the chickens. ${ }^{\text {a,b,c }}$ Different superscripts show the significance between groups at $p<0.05$. 

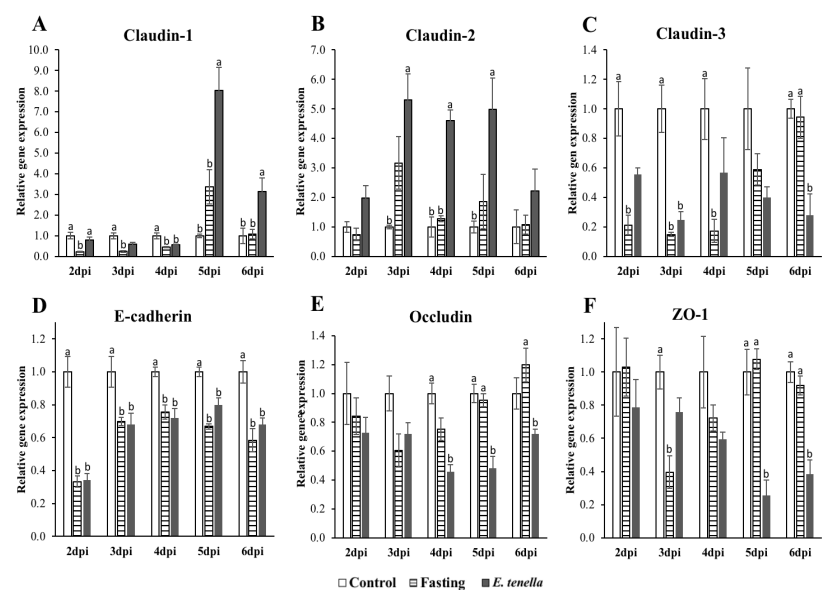

Fig 3. mRNA expression levels of junctional molecules in the ceca of chicken with $E$. tenella infection. ${ }^{\text {a,b }}$ Different superscripts show the significance between groups at $p<0.05$.

evidence indicated a close relation between OCDN and the integrity of the epithelial barrier.

E-cadherin molecules on the surfaces of epithelial cells are components of the adherens junctions ${ }^{7}$. In this experiment, we found that the mRNA expression level of E-cad was significantly downregulated from 2 to 6 dpi in response to E. tenella infection, which contributed to barrier dysfunction. During this period, merozoites might transmigrate along with epithelial cells and destroy the junction structure on the side, including E-cad. Similar to our results, previous studies have indicated that the infections of E. vermiformis ${ }^{13)}$ and Cryptosporidium parvum consequently resulted in the decreasing tendency of E-cad expression ${ }^{10)}$.

ZO-1, a membrane-associated guanylate kinase, has been reported to be critical for the formation of tight junction (CLDNs and OCDN) and the beltlike adherens junction complexes at cell-cell contacts ${ }^{14)}$. It is worth noting that the effects of ZO-1 on CLDNs and OCDN occur at the posttranslational level rather than the transcriptional level. From our results, we found that the expression level of ZO-1 in infected chicks was considerably decreased, reaching the bottom at 5 dpi. Depletion of ZO-1 obstructed the formation of junctional complexes, which might be linked to the diarrhea and bloody stool observed in $E$. tenella-infected chicks.

\section{CONCLUSION}

The disruption of barrier function via the downregulation of CLDN-1, CLDN-3, E-cad, OCDN, and ZO-1 and the upregulation of CLDN-2 might contribute to E. tenella infection induced diarrhea. The present findings might reveal that the expression levels of junctional molecule genes are related to the symptoms of $E$. tenella infection.
ACKNOWLEDGMENT: This research was supported by the Graduate School of Environmental and Life Science, Okayama University, under a Research Grant for Encouragement of Students.

\section{REFERENCES}

1) Chapman, H. D.: Milestones in avian coccidiosis research: A review, Poultry Science, Vol. 93, no. 3, pp. 501-511, 2014.

2) Lal, K., Bromley, E., Oakes, R., Prieto, J. H., Sanderson, S. J., Kurian, D., Hunt, L., Yates, J. R., Wastling, J. M., Sinden, R. E. and Tomley, F. M.: Proteomic comparison of four Eimeria tenella life-cycle stages: Unsporulated oocyst, sporulated oocyst, sporozoite and second-generation merozoite, Proteomics, Vol. 9, no. 19, pp. 4566-4576, 2009.

3) Groschwitz, K. R. and Hogan, S. P.: Intestinal Barrier Function: Molecular Regulation and Disease Pathogenesis, $J$ Allergy Clin Immunol, Vol 124, no. 1, pp. 3-22, 2009.

4) Erben, U., Loddenkemper, C., Doerfel, K., Spieckermann, S., Haller, D., Heimesaat, M. M., Zeitz, M., Siegmund, B. and Kühl, A. A.: A guide to histomorphological evaluation of intestinal inflammation in mouse models, Int $J$ Clin Exp Pathol, Vol. 7, no. 8, pp. 4557-4576, 2014.

5) Kuttappan, V. A., Berghman, L. R., Vicuna, E. A., Latorre, J. D., Menconi, A., Wolchok, J. D., Wolfenden, A. D., Faulkner, O. B., Tellez, G. I., Hargis, B. M. and Bielke, L. R.: Poultry enteric inflammation model with dextran sodium sulfate mediated chemical induction and feed restriction in broilers, Poult. Sci., Vol. 94, no. 6, pp. 1220-1226, 2015.

6) Livak, K. J. and Schmittgen, T. D.: Analysis of relative gene

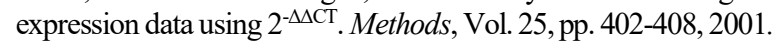

7) Lechuga, S. and Ivanov, A. I.: Disruption of the epithelial barrier during intestinal inflammation: Quest for new molecules and mechanisms, Molecular Cell Research, Vol. 1864, no. 7, pp. 1183-1194, 2017.

8) Van Itallie, C. M. and Anderson, J. M.: Claudins and epithelial paracellular transport, Annu. Rev. Physiol., Vol. 68, no. 1, pp. 403-429, 2006.

9) Chelakkot, C., Ghim, J. and Ryu, S. H.: Mechanisms regulating intestinal barrier integrity and its pathological implications, Exp. and Mol. Med., Vol. 50, no. 8, pp. 2-9, 2018.

10) Kumar, A., Chatterjee, I., Anbazhagan, A. N., Jayawardena, D., Priyamvada, S., Alrefai, W. A., Sun, J., Borthakur, A. and Dudeja, P. K.: Cryptosporidium parvum disrupts intestinal epithelial barrier function via altering expression of key tight junction and adherens junction proteins, Cell. Microbiol., Vol. 20, no. 6, pp. 1-13, 2018.

11) France, M. M. and Turner, J. R.: The mucosal barrier at a glance, J. Cell Sci., Vol. 130, no. 2, pp. 307-314, 2017.

12) Cani, P. D., Possemiers, S., Van De Wiele, T., Guiot, Y., Everard, A., Rottier, O., Geurts, L. and Naslain, D.: Changes in gut microbiota control inflammation in obese mice through a mechanism involving GLP-2-driven improvement of gut permeability, Gut, Vol. 58, no. 8, pp. 1091-1103, 2009.

13) Inagaki-Ohara, K., Dewi, F. N., Hisaeda, H., Smith, A. L., Jimi, F., Miyahira, M., Horii, Y. and Nawa, Y.: Intestinal intraepithelial lymphocytes sustain the epithelial barrier function against Eimeria vermiformis infection, Infect. Immun., Vol. 74, no. 9, pp. 5292-5301, 2006.

14) Ikenouchi, J., Umeda, K., Tsukita, S., Furuse, M. and Tsukita, S.: Requirement of ZO-1 for the formation of belt-like adherens junctions during epithelial cell polarization, J. Cell Biol., Vol. 176, no. 6, pp. 779-786, 2007.

(Received: January 29, 2021) 